\title{
ANÁLISIS MULTIVARIADO DE RECIDIVA Y PROGRESIÓN EN EL CARCINOMA DE CÉLULAS TRANSICIONALES DE VEJIGA EN ESTADIO T1. VALOR PRONÓSTICO DE p53 Y ki67
}

\author{
A. RODRÍGUEZ ALONSO*, S. PITA FERNÁNDEZ**, J. GONZÁLEZ-CARRERÓ***, \\ J.L. NOGUEIRA MARCH****
}

*Servicio de Urología. Hospital Arquitecto Marcide. El Ferrol (La Coruña). **Unidad de Epidemiología Clínica y Bioestadística. Hospital Juan Canalejo. La Coruña. Servicios de ***Anatomía Patológica y ****Urología. Hospital Xeral-Cíes. Vigo (Pontevedra).

Actas Urol Esp. 27 (2): 132-141, 2003

\section{RESUMEN}

“ANÁLISIS MULTIVARIADO DE RECIDIVA Y PROGRESIÓN EN EL CARCINOMA DE CÉLULAS TRANSICIONALES DE VEJIGA EN ESTADIO T1. VALOR PRONÓSTICO DE p53 Y ki67"

OBJETIVO: Establecer los factores pronósticos de recidiva y progresión en el carcinoma de células transicionales de vejiga en estadio T1, prestando especial atención al valor pronóstico de p53 y ki67.

MATERIAL Y MÉTODOS: 175 pacientes con tumor vesical incidente de la categoría T1. El estudio inmunohistoquímico fue realizado con los anticuerpos monoclonales DO7, para p53 y MIB-1, para ki67. Se utilizó la metodología de Kaplan-Meier y se realizó un análisis multivariado de regresión de Cox, para determinar variables asociadas a recidiva y progresión. Se realizaron asimismo curvas ROC para p53 y ki67.

RESULTADOS: El seguimiento medio fue de 8,55 años. La supervivencia libre de recidiva a los 5 y 12 años fue $57,98 \%$ y $48,47 \%$. Las variables independientes de recidiva fueron: grado histológico, multifocalidad tumoral, tamaño tumoral $>3 \mathrm{~cm}$, presencia de carcinoma in situ y expresión de ki67. La supervivencia libre de progresión a los 5 y 12 años fue 83,12\% y 75,63\%. Las variables independientes de progresión fueron: edad, multifocalidad tumoral, morfología microscópica sólida, expresión de p53 y expresión de ki67.

CONCLUSIONES: La morfología microscópica sólida y la expresión de p53 son las variables con mayor capacidad para predecir progresión tumoral. Se observa una correlación positiva entre la expresión de p53 y la progresión, de forma que cuanto mayor es la expresión de p53, mayor es la probabilidad de progresión. La multifocalidad tumoral y la expresión de ki67 >27\% son los principales factores pronósticos de recidiva.

PALABRAS CLAVE: p53. ki67. Neoplasia vesical. Recidiva. Progresión. Pronóstico.

\begin{abstract}
"MULTIVARIATE ANALYSIS OF RECURRENCE AND PROGRESSION IN STAGE T1 TRANSITIONAL CELL BLADDER CARCINOMA. PROGNOSTIC VALUE OF p53 AND ki67"

OBJECTIVE: To establish prognostic factors of recurrence and progression in stage T1 transitional cell bladder carcinoma, paying special attention to prognostic value of p53 and ki67.

MATERIALS AND METHODS: 175 patients with incident bladder tumour at T1 category. The immunohistochemical study was performed using the monoclonal antibodies DO-7, for p53 and MIB-1, for ki67. Kaplan-Meier methodology was used, and a multivariate analysis using Cox's proportional hazards model was carried out in order to determine the variables associated with recurrence and progression. ROC curves were also drawn up.

RESULTS: The average follow up period was 8.55 years. 5 and 12 -year recurrence-free survival rates were $57.98 \%$ and $48.47 \%$. The independent variables for recurrence were histological grade, tumour multifocality, tumour size $>3 \mathrm{~cm}$, presence of carcinoma in situ and ki67 expression. 5 and 12-year progression-free survival rates were $83.12 \%$ and $75.63 \%$. The independent variables for progression were age, tumour multifocality, solid microscopic morphology, p53 expression and ki67 expression.

CONCLUSIONS: Solid microscopic pattern and p53 expression are the variables which best predict progression. A positive relationship was observed between p53 and progression: the greater the expression of p53, the greater the progression. Tumour multifocality and ki67 expression $>27 \%$ are the main prognostic factors for recurrence.
\end{abstract}

KEY WORDS: p53. ki67. Bladder neoplasm. Recurrence. Progression. Prognosis. 
$\mathrm{L}^{\mathrm{s} s}$ a categoría $\mathrm{T}$ y el grado histológico del tumor constituyen los parámetros usados tradicionalmente para predecir el pronóstico de los tumores superficiales de vejiga. Sin embargo, la existencia de una variabilidad intra e interobservador de aproximadamente el 50\% en la determinación del grado histológico ${ }^{1}$, y el elevado porcentaje de supraestadificación patológica, que es superior al $25 \%$ en la mayoría de las series de cistectomía radical $^{2}$, reflejan las limitaciones de ambos parámetros en la predicción del pronóstico del carcinoma de células transicionales superficial de vejiga.

El gen p53 mutado transcribe una proteína que es metabólicamente más estable que la proteína p53 nativa. Esto hace posible su detección mediante métodos inmunohistoquímicos convencionales $^{3}$.

$\mathrm{El}$ anticuerpo monoclonal ki67, que fue inicialmente empleado para el estudio inmunohistoquímico, tenía el inconveniente de que sólo podía utilizarse con secciones tisulares frescas congeladas, ya que el epitopo del antígeno al que se unía, sufría una degeneración cuando el tejido era fijado, parafinado y embebido. La reciente caracterización de la proteína identificada por el anticuerpo ki67, ha facilitado el desarrollo del anticuerpo monoclonal MIB-1, que permite el estudio inmunohistoquímico de muestras tisulares fijadas con formol e incluidas en parafina, posibilitando de este modo la realización de estudios retrospectivos.

El propósito del presente estudio es determinar cuales son los factores pronósticos relacionados con la recidiva y la progresión del carcinoma de células transicionales (CCT) de vejiga en estadio $\mathrm{T} 1$, prestando especial atención al valor pronóstico de p53 y ki67.

\section{MATERIAL Y MÉTODOS}

Se realizó un estudio observacional de cohorte retrospectivo y prospectivo, incluyendo 175 pacientes con CCT de vejiga en estadio T1, diagnosticados entre enero de 1987 y diciembre de 1992 en el Complejo Hospitalario Xeral-Cíes de Vigo. Todos los tumores incluidos en el estudio eran incidentes.

En cada uno de los casos se analizaron 177 variables, siendo las más relevantes: sexo, edad, hábito tabáquico, síntomas de presentación, exis- tencia de carcinoma in situ (CIS) en la biopsia vesical randomizada, presencia de CIS en la uretra prostática, grado histológico, número de tumores, tamaño del tumor más grande, aspecto endoscópico-macroscópico del tumor, patrón de crecimiento microscópico, tamaño del núcleo celular, porcentaje de expresión nuclear de p53 y porcentaje de expresión nuclear de ki67.

Se definió recidiva como la aparición de un nuevo tumor de estirpe urotelial en cualquier localización del tracto urinario. Se definió progresión como el incremento de las categorías $\mathrm{T}$, $\mathrm{N}$ ó $\mathrm{M}$, en cualquiera de las recidivas.

Todos los tumores incidentes fueron tratados mediante resección transuretral (RTU) hasta pericisto, con biopsia del lecho tumoral. Ningún paciente fue sometido a tratamiento para el tumor vesical, previo a la RTU. Todos los pacientes recibieron algún tipo de tratamiento quimio o inmunoterápico intravesical tras la RTU del tumor primario. Ningún paciente fue sometido a tratamiento sistémico neoadyuvante o adyuvante.

Seguimiento de los pacientes: Se realizó del siguiente modo: 1) Cistoscopia trimestral durante el primer año, cistoscopia semestral el segundo año, y cistoscopia anual a partir del tercer año. En aquellos pacientes con intolerancia a la cistoscopia, el seguimiento se realizó mediante ecografía vesical, siguiendo la misma pauta de revisión; 2) Citología de orina y estudio de la función renal mediante cifras sanguíneas de urea y creatinina, cada seis meses durante los dos primeros años, y posteriormente cada año; 3) Sedimento de orina semestral durante los primeros dos años, y anual a partir del tercer año; 4) Urografía intravenosa anual, los dos primeros años, y posteriormente bianual.

Evaluación anatomopatológica: La graduación de los tumores del estudio se efectuó mediante la escala de la Organización Mundial de la Salud (OMS) de 1973, que divide el CCT en tres grados ${ }^{4}$. La estadificación patológica se realizó de acuerdo con los criterios de la American Joint Cancer Committee/Union Internationale Contre le Cancer (AJCC/UICC) publicados en $1997^{5}$.

Estudio inmunohistoquímico: Se examinaron microscópicamente todas las preparaciones originales teñidas con Hematoxilina-Eosina, seleccionando una de cada caso para efectuar el estudio 
inmunohistoquímico. La selección se hizo atendiendo exclusivamente a la cantidad de tumor presente y a la aparente viabilidad morfológica de las células tumorales. Del bloque de parafina seleccionado se obtuvieron secciones de 4 micras. Dichas secciones fueron desparafinadas comenzando con su incubación en estufa a $60^{\circ} \mathrm{C}$ durante 12 horas, seguida de pases a través de xilol y etanol a concentraciones decrecientes, hasta su completa rehidratación, llegando a agua destilada. Posteriormente y de forma sistemática se realizó una recuperación o desenmascaramiento de antígenos, calentando en olla a presión las preparaciones sumergidas en buffer Citrato $10 \mathrm{mM}(\mathrm{pH}$ 6) o buffer EDTA $1 \mathrm{mM}$ (pH 8), durante 2-3 minutos a plena presión. La tinción inmunohistoquímica se efectuó mediante un sistema automatizado denominado Ventana NexES (Ventana Medical Systems, Inc., Tucson, Arizona, EEUU). Este sistema, tras un paso de desactivación de la peroxidasa endógena, dosifica el anticuerpo primario. Los anticuerpos monoclonales utilizados en este estudio fueron: proteína p53, clon DO7, Novocastra Laboratories, Newcastle, Reino Unido (referencia NCL-p53-DO7), dilución 1:25. Antígeno ki67, clon MIB-1, Immunotech, Marsella, Francia (referencia PN IM0505), dilución $1: 100$. Estos anticuerpos primarios fueron incubados durante 30 minutos a $40^{\circ} \mathrm{C}$.

Posteriormente el sistema revela la reacción inmunológica en el tejido mediante la técnica de la Avidina-Biotina-Peroxidasa, utilizando como cromógeno la Diaminobenzina, que precipita en el tejido, dando lugar a un pigmento parduzco estable e insoluble en disolventes orgánicos. Las preparaciones fueron contrateñidas con Hematoxilina y montadas por el procedimiento habitual.

La evaluación cuantitativa y cualitativa fue realizada por un solo observador, utilizando un microscopio óptico Olympus BX40. El porcentaje de expresión nuclear de p53 y ki67 fue determinado calculando la media de los cuatro campos microscópicos de gran aumento (40X) con mayor número de núcleos teñidos. En todos los casos se contabilizaron más de 3000 núcleos celulares.

Análisis estadístico: Se realizó un estudio descriptivo de todas las variables con su 95\% intervalo de confianza (IC). Las variables cuantitativas se expresan con sus medidas de centralización y dispersión, utilizando en algunos casos la categorización de las mismas en función de su mediana o cuartiles. Se realizó un análisis de supervivencia mediante la metodología Kaplan-Meier, siendo las variables dependientes de interés: recidiva y progresión. Para detectar diferencias entre los distintos estratos de las variables, se utilizó el test de Log-rank. Para determinar como la recidiva y la progresión se ven afectadas por otras covariables, y para controlar por el efecto confusor de las mismas, se realizó un análisis multivariado de regresión de Cox. Los riesgos fueron estimados con su 95\%IC. Se calculó asimismo el área bajo la curva ROC, para predecir las probabilidades de recidiva y progresión, respecto a los valores de p53 y ki67. El análisis estadístico se realizó con el programa SPSS 10.0 para Windows.

\section{RESULTADOS}

La media de edad de los pacientes fue de $66 \pm$ 12,01 años. El 84\% de ellos eran hombres. El seguimiento medio fue de 102,64 meses $(8,55$ años). 20 pacientes tenían tumores G1 (11,43\%), 121 tumores eran G2 $(69,14 \%)$, y 34 eran G3 $(19,43 \%)$. Las características epidemiológicas, clínicas y patológicas más relevantes de los pacientes pueden observarse en la Tabla I.

Durante el seguimiento se produjeron 72 muertes, siendo el cáncer de vejiga la causa principal de las mismas $(36,11 \%)$.

\section{Recidiva}

La supervivencia libre de recidiva al cabo de 5 y 12 años fue de 57,98\% y 48,47\%, respectivamente.

Las variables significativamente asociadas con la recidiva en el análisis univariado fueron: síntomas iniciales de presentación, multifocalidad tumoral, CIS en la biopsia vesical randomizada, y expresión nuclear de p53 en cuartiles (Tabla II). La existencia de tumores múltiples, CIS en la biopsia randomizada de vejiga, síntomas miccionales irritativos y un porcentaje elevado de expresión de p53, se asociaron a una probabilidad significativamente aumentada de recidiva (Figs. 1 y 2).

Se realizó un análisis multivariado de regresión de Cox, ajustando por las siguientes covariables: sexo, edad, grado histológico, multifocalidad tumoral, tamaño tumoral, aspecto endoscópico, patrón 
TABLA I

\section{CARACTERÍSTICAS CLÍNICAS Y PATOLÓGICAS DE LOS PACIENTES DEL ESTUDIO}

\begin{tabular}{|c|c|c|c|}
\hline Variable & & Frec (\%) & 95\% I.C. \\
\hline Sexo & $\begin{array}{l}\text { Hombre } \\
\text { Mujer }\end{array}$ & $\begin{array}{l}147(84,00) \\
28(16,00)\end{array}$ & $\begin{array}{l}78,00-88,88 \\
11,12-21,99\end{array}$ \\
\hline Tabaquismo & $\begin{array}{l}\text { Fumador } \\
\text { No fumador }\end{array}$ & $\begin{array}{c}128(73,14) \\
47(26,86)\end{array}$ & $\begin{array}{l}66,21-79,31 \\
20,68-33,79\end{array}$ \\
\hline Sintomas iniciales & $\begin{array}{l}\text { Macrohematuria } \\
\text { Sintomas irritativos } \\
\text { Desconocidos }\end{array}$ & $\begin{array}{c}136(77,71) \\
30(17,14) \\
9(5,14)\end{array}$ & $\begin{array}{c}71,10-83,42 \\
12,09-23,27 \\
2,53-9,22\end{array}$ \\
\hline $\begin{array}{l}\text { Tiempo desde los sintomas } \\
\text { iniciales hasta el diagnóstico }\end{array}$ & $\begin{array}{l}\leq 6 \text { meses } \\
>6 \text { meses } \\
\text { Desconocido }\end{array}$ & $\begin{array}{l}103(58,86) \\
30(17,14) \\
42(24,00)\end{array}$ & $\begin{array}{l}51,45-65,97 \\
12,09-23,27 \\
18,11-30,74\end{array}$ \\
\hline Número de tumores & $\begin{array}{l}\text { Tumor solitario } \\
\text { Tumor múltiple } \\
\text { Desconocido }\end{array}$ & $\begin{array}{c}108(61,71) \\
65(37,14) \\
2(1,14)\end{array}$ & $\begin{array}{c}54,34-68,70 \\
30,22-44,48 \\
0,19-3,72\end{array}$ \\
\hline Tamaño tumoral & $\begin{array}{l}\leq 3 \mathrm{~cm} \\
>3 \mathrm{~cm} \\
\text { Desconocido }\end{array}$ & $\begin{aligned} 99 & (56,57) \\
72 & (41,14) \\
4 & (2,28)\end{aligned}$ & $\begin{array}{c}49,15-63,78 \\
34,02-48,55 \\
0,73-5,42\end{array}$ \\
\hline $\begin{array}{l}\text { Aspecto endoscópico del } \\
\text { tumor }\end{array}$ & $\begin{array}{l}\text { Papilar } \\
\text { Sólido } \\
\text { Desconocido }\end{array}$ & $\begin{array}{c}158(90,28) \\
8(4,57) \\
9(5,14)\end{array}$ & $\begin{array}{c}85,20-94,04 \\
2,14-8,49 \\
2,53-9,22\end{array}$ \\
\hline $\begin{array}{l}\text { Patrón de crecimiento } \\
\text { microscópico }\end{array}$ & $\begin{array}{l}\text { Papilar } \\
\text { Sólido } \\
\text { Desconocido }\end{array}$ & $\begin{array}{c}168(96,00) \\
6(3,43) \\
1(0,57)\end{array}$ & $\begin{array}{c}92,25-98,23 \\
1,40-6,99 \\
0,03-2,78\end{array}$ \\
\hline $\begin{array}{l}\text { Correspondencia } \\
\text { macro-microscópica }\end{array}$ & $\begin{array}{l}\text { Sí correspondencia } \\
\text { No correspondencia } \\
\text { Desconocida }\end{array}$ & $\begin{array}{c}157(89,71) \\
9(5,14) \\
9(5,14)\end{array}$ & $\begin{array}{c}84,53-93,59 \\
2,53-9,22 \\
2,53-9,22\end{array}$ \\
\hline Hidronefrosis & $\begin{array}{l}\text { Unilateral } \\
\text { Bilateral } \\
\text { No hidronefrosis }\end{array}$ & $\begin{array}{c}6(3,43) \\
1(0,57) \\
168(96,00)\end{array}$ & $\begin{array}{c}1,40-6,99 \\
0,03-2,78 \\
92,25-98,23\end{array}$ \\
\hline $\begin{array}{l}\text { Localización intravesical } \\
\text { del tumor }\end{array}$ & $\begin{array}{l}\text { Cara lateral izquierda } \\
\text { Trígono } \\
\text { Cara lateral derecha } \\
\text { Cara posterior } \\
\text { Cara anterior } \\
\text { Cuello vesical } \\
\text { Fondo-cúpula }\end{array}$ & $\begin{array}{c}74(42,28) \\
72(41,14) \\
68(38,86) \\
55(31,42) \\
32(18,28) \\
30(17,14) \\
7(4,00)\end{array}$ & $\begin{array}{c}35,12-49,70 \\
34,02-48,55 \\
31,84-46,23 \\
24,87-38,59 \\
13,08-24,53 \\
12,09-23,27 \\
1,76-7,75\end{array}$ \\
\hline $\begin{array}{l}\text { Biopsia vesical } \\
\text { randomizada }\end{array}$ & $\begin{array}{l}\text { Positiva (CIS) } \\
\text { Negativa (No CIS) } \\
\text { No realizada }\end{array}$ & $\begin{array}{c}8(4,57) \\
160(91,43) \\
7(4,00)\end{array}$ & $\begin{array}{c}2,14-8,49 \\
86,56-94,93 \\
1,76-7,75\end{array}$ \\
\hline $\begin{array}{l}\text { Estado de la uretra } \\
\text { prostática }\end{array}$ & $\begin{array}{l}\text { CIS } \\
\text { No Cis } \\
\text { Desconocido }\end{array}$ & $\begin{array}{c}2(1,36) \\
84(57,14) \\
61(41,49)\end{array}$ & $\begin{array}{c}0,23-4,42 \\
49,04-64,96 \\
33,73-49,59\end{array}$ \\
\hline Grado histológico & $\begin{array}{l}\text { G1 } \\
\text { G2 } \\
\text { G3 }\end{array}$ & $\begin{array}{c}20(11,43) \\
121(69,29) \\
34(19,43)\end{array}$ & $\begin{array}{r}7,32-16,80 \\
62,00-75,65 \\
14,07-25,79\end{array}$ \\
\hline
\end{tabular}


TABLA II

VARIABLES QUE MODIFICAN SIGNIFICATIVAMENTE LA SUPERVIVENCIA LIBRE DE RECIDIVA

\begin{tabular}{|c|c|c|c|c|c|}
\hline Variable & & $\begin{array}{l}\text { Supervivencia } \\
\text { libre de recidiva } \\
\text { a los } 5 \text { años }\end{array}$ & $\begin{array}{l}\text { Supervivencia } \\
\text { libre de recidiva } \\
\text { a los } 12 \text { años }\end{array}$ & Test Log-rank & $\mathbf{p}$ \\
\hline $\begin{array}{l}\text { Multifocalidad } \\
\text { tumoral }\end{array}$ & $\begin{array}{l}\text { Tumor solitario } \\
\text { Tumor múltiple }\end{array}$ & $\begin{array}{l}71,45 \% \\
38,87 \%\end{array}$ & $\begin{array}{l}61,85 \% \\
28,02 \%\end{array}$ & 17,34 & 0,0000 \\
\hline Síntomas iniciales & $\begin{array}{l}\text { Macrohematuria } \\
\text { Otros síntomas }\end{array}$ & $\begin{array}{l}63,03 \% \\
33,33 \%\end{array}$ & $\begin{array}{l}51,52 \% \\
33,33 \%\end{array}$ & 5,74 & 0,0166 \\
\hline $\begin{array}{l}\text { Biopsia vesical } \\
\text { randomizada }\end{array}$ & $\begin{array}{l}\text { No CIS } \\
\text { CIS }\end{array}$ & $\begin{array}{l}59,60 \% \\
28,57 \%\end{array}$ & $\begin{array}{c}50,88 \% \\
00,00 \% *\end{array}$ & 7,41 & 0,0065 \\
\hline $\begin{array}{l}\text { Expresión de p53 } \\
\text { en cuartiles }\end{array}$ & $\begin{array}{l}<0,2 \% \\
0,2-2 \% \\
2-14 \% \\
>14 \%\end{array}$ & $\begin{array}{l}71,87 \% \\
59,48 \% \\
55,80 \% \\
39,36 \%\end{array}$ & $\begin{array}{l}64,94 \% \\
48,33 \% \\
45,40 \% \\
30,36 \%\end{array}$ & 11,91 & 0,0077 \\
\hline
\end{tabular}

*: Supervivencia libre de recidiva a los 7 años.

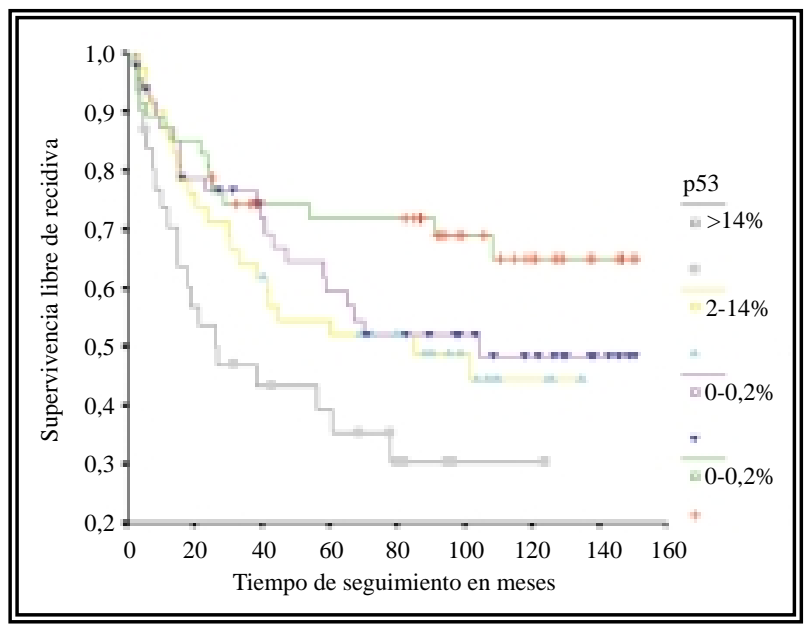

FIGURA 1. Probabilidad de recidiva del CCT de vejiga en estadio $\mathrm{T} 1$ en función de la expresión de p53 en cuartiles (test $\log$ rank $=11,91 ; p=0,0077$ ).

de crecimiento microscópico, presencia de CIS en la biopsia vesical randomizada, expresión nuclear de p53 en cuartiles y expresión nuclear de ki67 en cuartiles (Tabla III).

Las variables asociadas con la recidiva de forma independiente fueron: grado histológico, multifocalidad tumoral, tamaño tumoral $>3 \mathrm{~cm}$, CIS en biopsia vesical randomizada y expresión de ki67 >27\%.

\section{Progresión}

La supervivencia libre de progresión a los 5 y 12 años fue de $83,12 \%$ y $75,63 \%$, respectivamente.

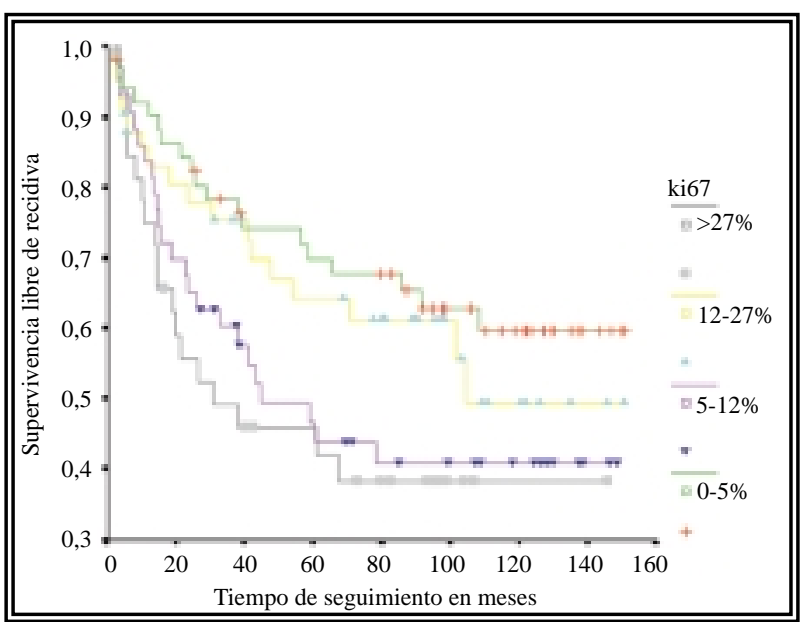

FIGURA 2. Probabilidad de recidiva del CCT de vejiga en estadio 11 en función de la expresión de ki67 en cuartiles (test $\log$ rank $=7,80 ; p=0,0503$ ).

Las variables que modificaron significativamente la progresión en el análisis univariado fueron las siguientes: multifocalidad tumoral, aspecto endoscópico de la lesión, patrón de crecimiento microscópico, CIS en la biopsia randomizada, expresión de p53 en cuartiles y expresión de ki67 en cuartiles (Tabla IV). La presencia de múltiples tumores, la morfología macroscópica y microscópica sólida, CIS en la biopsia randomizada, y un porcentaje elevado de expresión nuclear de p53 y ki67, se asociaron a una probabilidad significativamente elevada de progresión (Figs. 3 y 4). 
TABLA III

ANÁLISIS DE REGRESIÓN DE COX PARA PREDECIR RECIDIVA

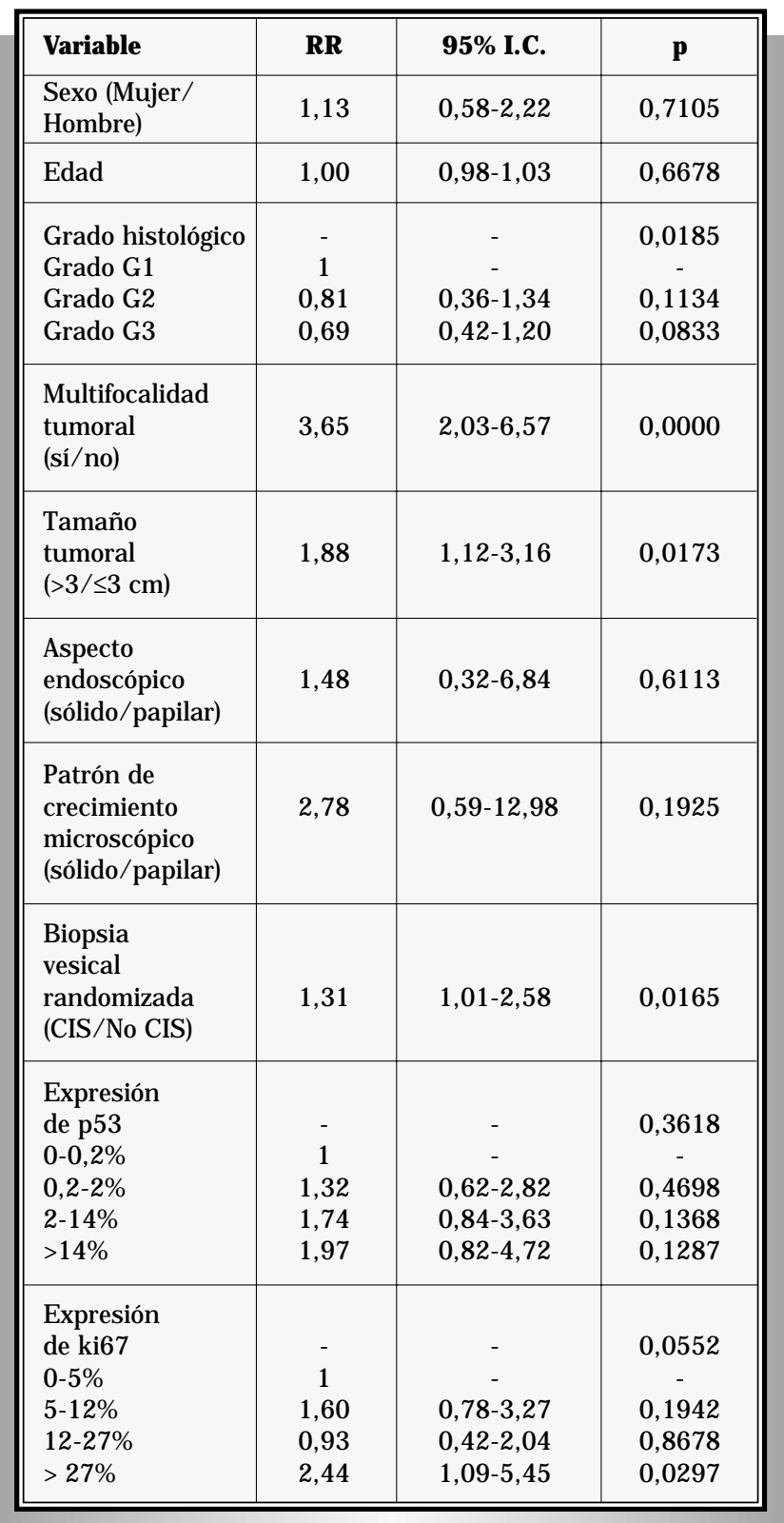

Se efectuó asimismo un análisis multivariado de riesgos proporcionales de Cox, controlando por una serie de variables: sexo, edad, grado histológico, multifocalidad tumoral, tamaño tumoral, aspecto endoscópico, patrón de crecimiento microscópico, expresión de p53 en cuartiles y expresión de ki67 en cuartiles (Tabla V). En dicho análisis se observó que las variables con capacidad para predecir la progresión de forma independiente eran: edad, multifocalidad tumoral, patrón de crecimiento microscópico sólido, expresión de p53 y expresión de ki67. Las expresiones de p53 y ki67 mostraron una relación con la progresión que podríamos llamar dosis-respuesta, de forma que cuanto mayor era el porcentaje de expresión inmunohistoquímica de p53 y ki67, mayor era el riesgo de progresión tumoral.

Las curvas ROC de recidiva y progresión para las variables p53 y ki67, y el área bajo estas curvas se encuentran representadas en las Figs. 5 y 6. Se puede observar que, de forma general, la expresión de p53 muestra una mayor capacidad para predecir el pronóstico que la expresión de ki67.

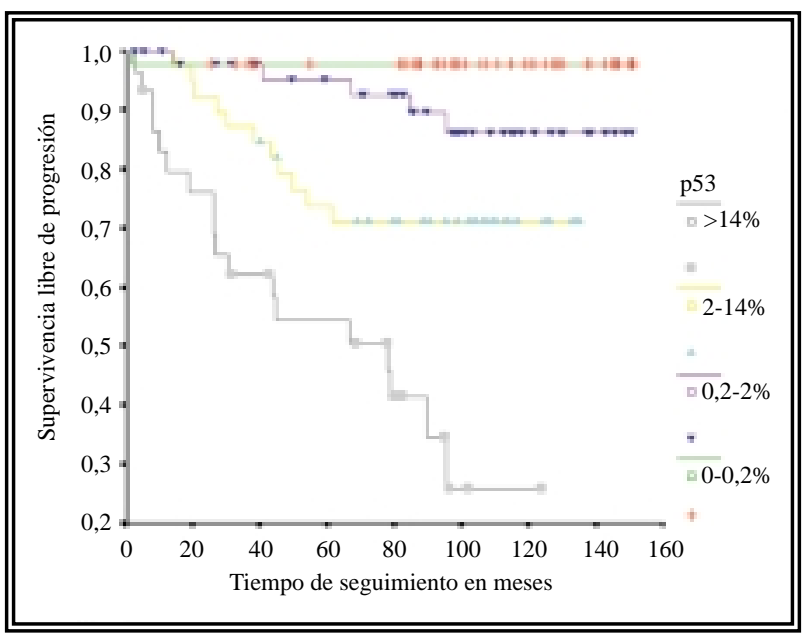

FIGURA 3. Probabilidad de progresión del CCT de vejiga en estadio T1 en función de la expresión de p53 en cuartiles (test $\log$ rank $=53,04 ; p=0,0000$ ).

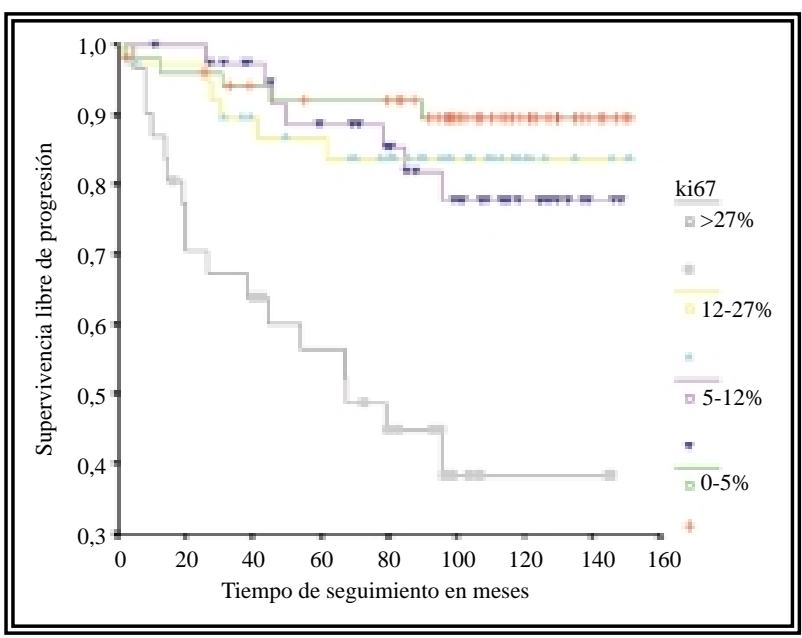

FIGURA 4. Probabilidad de progresión del CCT de vejiga en estadio T1 en función de la expresión de ki67 en cuartiles (test $\log$ rank $=34,03 ; p=0,0000$ ). 
VARIABLES QUE MODIFICAN SIGNIFICATIVAMENTE LA SUPERVIVENCIA LIBRE DE PROGRESIÓN

\begin{tabular}{|c|c|c|c|c|c|}
\hline Variable & & $\begin{array}{c}\text { Supervivencia } \\
\text { libre de } \\
\text { progresión } \\
\text { a los } 5 \text { años }\end{array}$ & $\begin{array}{c}\text { Supervivencia } \\
\text { libre de } \\
\text { progresión } \\
\text { a los } 12 \text { años }\end{array}$ & Test Log-rank & $\mathbf{p}$ \\
\hline $\begin{array}{l}\text { Multifocalidad } \\
\text { tumoral }\end{array}$ & $\begin{array}{l}\text { Tumor solitario } \\
\text { Tumor múltiple }\end{array}$ & $\begin{array}{l}90,37 \% \\
72,24 \%\end{array}$ & $\begin{array}{l}83,91 \% \\
64,13 \%\end{array}$ & 7,71 & 0,0055 \\
\hline Aspecto endoscópico & $\begin{array}{l}\text { Papilar } \\
\text { Sólido }\end{array}$ & $\begin{array}{l}84,81 \% \\
58,33 \%\end{array}$ & $\begin{array}{l}78,23 \% \\
58,33 \%\end{array}$ & 4,08 & 0,0435 \\
\hline $\begin{array}{l}\text { Patrón de crecimiento } \\
\text { microscópico }\end{array}$ & $\begin{array}{l}\text { Papilar } \\
\text { Sólido }\end{array}$ & $\begin{array}{l}84,93 \% \\
50,00 \%\end{array}$ & $\begin{array}{l}77,84 \% \\
33,33 \%\end{array}$ & 12,57 & 0,0004 \\
\hline $\begin{array}{l}\text { Biopsia vesical } \\
\text { randomizada }\end{array}$ & $\begin{array}{l}\text { No CIS } \\
\text { CIS }\end{array}$ & $\begin{array}{l}84,35 \% \\
57,14 \%\end{array}$ & $\begin{array}{l}77,92 \% \\
19,05 \%\end{array}$ & 11,62 & 0,0007 \\
\hline $\begin{array}{l}\text { Expresión de p53 } \\
\text { en cuartiles }\end{array}$ & $\begin{array}{l}\leq 0,2 \% \\
0,2-2 \% \\
2-14 \% \\
>14 \%\end{array}$ & $\begin{array}{l}97,83 \% \\
95,27 \% \\
73,77 \% \\
54,44 \%\end{array}$ & $\begin{array}{l}97,83 \% \\
86,09 \% \\
71,04 \% \\
25,85 \%\end{array}$ & 53,04 & 0,0000 \\
\hline $\begin{array}{l}\text { Expresión de ki67 } \\
\text { en cuartiles }\end{array}$ & $\begin{array}{l}\leq 5 \% \\
5-12 \% \\
12-27 \% \\
>27 \%\end{array}$ & $\begin{array}{l}92,02 \% \\
88,66 \% \\
86,70 \% \\
56,33 \%\end{array}$ & $\begin{array}{l}89,53 \% \\
77,73 \% \\
83,71 \% \\
38,36 \%\end{array}$ & 34,03 & 0,0000 \\
\hline
\end{tabular}

\section{DISCUSION}

Aproximadamente el $50-80 \%$ de los tumores superficiales de vejiga presentarán recidiva en algún momento de su evolución ${ }^{6,7}$. Aunque la mayoría de las recidivas (70-80\%) se diagnostican durante los primeros 18 meses tras la RTU del tumor inicial ${ }^{6,8}$, más del $34 \%$ de los pacientes presentarán su primera recidiva después del tercer año de seguimiento, según observaron Ojea Calvo et $\mathrm{al}^{9}$. El seguimiento medio de los pacientes de nuestro estudio fue de 8,55 años, y el 20\% de las recidivas tuvieron lugar a partir del tercer año de seguimiento. Por ello, creemos que es absolutamente necesario un largo periodo de seguimiento para poder analizar los factores pronósticos asociados al cáncer de vejiga, teniendo en cuenta la peculiar naturaleza de esta enfermedad.

La multifocalidad tumoral se mostró en nuestro estudio como la variable independiente con mayor asociación con la recidiva tumoral, de forma que los tumores múltiples tenían un riesgo 3,65 veces superior de recidivar que los tumores solitarios. La existencia de multifocalidad tumoral es uno de los factores de riesgo más estrechamente asociados a recidiva en la cavidad vesi- cal, según demuestran Millán-Rodríguez et al ${ }^{10}$, Kiemeney et $\mathrm{al}^{11}$, Witjes et $\mathrm{al}^{1,7}$ y Allard et $\mathrm{al}^{6}$; constituyendo asimismo el principal factor de riesgo de recidiva en el tracto urinario superior, según observan Millán-Rodríguez et $\mathrm{al}^{12}$. La multifocalidad tumoral representa también uno de los principales factores pronósticos de progresión tumoral, como se demuestra en nuestro estudio $\mathrm{y}$ en otros similares ${ }^{10,11,13}$.

Algunos autores consideran que el grado histológico es un parámetro importante en la predicción de recidiva ${ }^{6,7,14}$, mientras que otros no encuentran relación alguna ${ }^{1,10,11}$. Nuestro estudio muestra que el grado histológico es una variable independiente de recidiva, aunque paradójicamente, los tumores con mayor probabilidad de recidiva son los de grado 1. Estos resultados son probablemente una consecuencia de la enorme subjetividad existente en la determinación del grado histológico, que da lugar a una variabilidad intra e interobservador de aproximadamente el $50 \%{ }^{1,14}$.

En nuestro estudio, la presencia de CIS en la biopsia vesical randomizada se comporta como una variable independiente de recidiva. Millán- 
TABLA V

\begin{tabular}{|c|c|c|c|}
\hline Variable & $\mathbf{R R}$ & 95\% I.C. & $\mathbf{p}$ \\
\hline $\begin{array}{l}\text { Sexo (Mujer/ } \\
\text { Hombre) }\end{array}$ & 1,56 & $0,42-5,86$ & 0,5072 \\
\hline Edad & 1,06 & $1,00-1,12$ & 0,0333 \\
\hline $\begin{array}{l}\text { Grado histológico } \\
\text { Grado G1 } \\
\text { Grado G2 } \\
\text { Grado G3 }\end{array}$ & $\begin{array}{c}- \\
1 \\
1,02 \\
1,09\end{array}$ & $\begin{array}{c}- \\
- \\
0,54-1,16 \\
0,70-1,23\end{array}$ & $\begin{array}{c}0,0577 \\
- \\
0,0877 \\
0,0642\end{array}$ \\
\hline $\begin{array}{l}\text { Multifocalidad } \\
\text { tumoral } \\
\text { (síno) }\end{array}$ & 3,10 & $1,25-7,67$ & 0,0145 \\
\hline $\begin{array}{l}\text { Tamaño } \\
\text { tumoral } \\
(>3 / \leq 3 \mathrm{~cm})\end{array}$ & 1,92 & $0,87-4,23$ & 0,1042 \\
\hline $\begin{array}{l}\text { Aspecto } \\
\text { endoscópico } \\
\text { (sólido/papilar) }\end{array}$ & 3,78 & $0,65-21,85$ & 0,1368 \\
\hline $\begin{array}{l}\text { Patrón de } \\
\text { crecimiento } \\
\text { microscópico } \\
\text { (sólido/papilar) }\end{array}$ & 55,19 & $8,22-370,65$ & 0,0000 \\
\hline $\begin{array}{l}\text { Expresión } \\
\text { de p53 } \\
0-0,2 \% \\
0,2-2 \% \\
2-14 \% \\
>14 \%\end{array}$ & $\begin{array}{c}- \\
1 \\
11,21 \\
20,18 \\
74,21\end{array}$ & $\begin{array}{c}- \\
- \\
1,21-104,00 \\
2,27-179,22 \\
7,87-699,58\end{array}$ & $\begin{array}{c}0,0002 \\
- \\
0,0334 \\
0,0070 \\
0,0002\end{array}$ \\
\hline $\begin{array}{l}\text { Expresión } \\
\text { de ki67 } \\
0-5 \% \\
5-12 \% \\
12-27 \% \\
>27 \%\end{array}$ & $\begin{array}{c}- \\
1 \\
1,17 \\
1,16 \\
5,29\end{array}$ & $\begin{array}{c}- \\
- \\
0,30-4,50 \\
0,27-4,94 \\
1,48-18,84\end{array}$ & $\begin{array}{c}0,0040 \\
- \\
0,8180 \\
0,8350 \\
0,0102\end{array}$ \\
\hline
\end{tabular}

Rodríguez et al observan que la positividad de la biopsia aleatoria es una variable independiente en la predicción de recidiva ${ }^{10}$. Kiemeney et $\mathrm{al}^{11}$, por el contrario, no encuentran una relación significativa entre el resultado positivo de la biopsia vesical y la recidiva tumoral. Entre estos dos estudios existen importantes diferencias metodológicas, tanto en el número de biopsias realizadas, como en la técnica utilizada para la toma de biopsias, e incluso en la definición de biopsia positiva. En nuestro trabajo, se tomaron 5 muestras biópsicas de mucosa endoscópicamente normal (incluyendo una biopsia de uretra prostática), mediante una pinza fría flexible, excepto la biopsia de la uretra prostática, que fue realizada con el asa del resector. Siguiendo las recomendaciones de la OMS y la International Society of Urological Pathology (ISUP), solamente aquellas biopsias con CIS fueron consideradas como positivas ${ }^{15}$.

Existen muy pocos estudios en los que se analice de forma multivariada la importancia de la expresión inmunohistoquímica de ki67 en la recidiva de los tumores vesicales superficiales, y los resultados de los mismos no son coincidentes. Así, mientras Gontero et al observan que la expresión de ki67 tiene una capacidad pronóstica independiente de recidiva ${ }^{16}$, Liukkonen et $\mathrm{al}^{14}$ y Zlotta et $\mathrm{al}^{17}$, no encuentran una relación significativa. Por otra parte, Pfister et al observan que la expresión de ki67 se relaciona de forma significativa con la recidiva, sólo en los tumores mayores de $3 \mathrm{~cm}^{8}$.

Los resultados de nuestro estudio ponen de manifiesto que la expresión de ki67 >27\% es una variable independiente de recidiva. Es por ello posible que los tumores de la categoría $\mathrm{T} 1$, con una elevada expresión de ki67 deban considerarse como tumores de alto riesgo de recidiva, y ser tratados como tales, como ocurre en el caso de los tumores multifocales, los tumores mayores de $3 \mathrm{~cm}$, o aquellos con CIS asociado; variables estas últimas cuya independencia en la predicción de recidiva también se demuestra en nuestro estudio.

La progresión tumoral es un momento de especial importancia en los tumores de la categoría $\mathrm{T} 1$, ya que el desarrollo de infiltración de la capa muscular propia implica un cambio drástico en la orientación terapéutica y en el pronóstico del paciente. En nuestro estudio observamos que el riesgo de progresión de los tumores microscópicamente sólidos es 55 veces superior al de los tumores papilares. Liukkonen et al observan que la morfología papilar del tumor ejerce un efecto protector, con un riesgo de progresión papilar/sólido de $0,31^{14}$. El patrón de crecimiento microscópico del tumor es una variable que no se analiza en la mayoría de los estudios multivariados de progresión, aún cuando constituye un parámetro fácilmente medible, con una escasa carga de subjetividad $^{10,11,13,17-19}$.

La expresión de ki67 se halla estrechamente vinculada a la fracción de crecimiento tumoral, de 


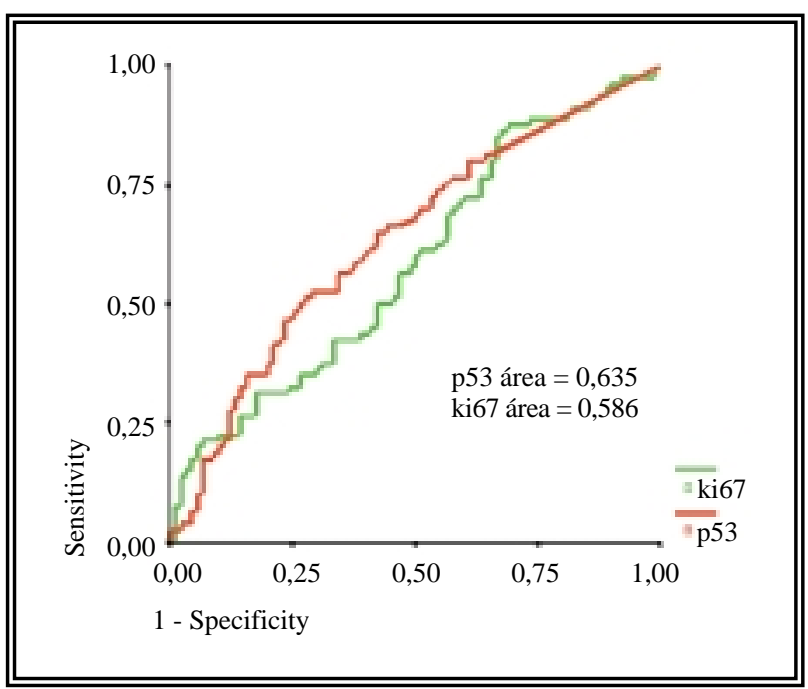

FIGURA 5. Curvas ROC de p53 y ki67 para la recidiva (área p53: 0,635 \pm 0,043; área ki67: 0,596 $\pm 0,044)$.

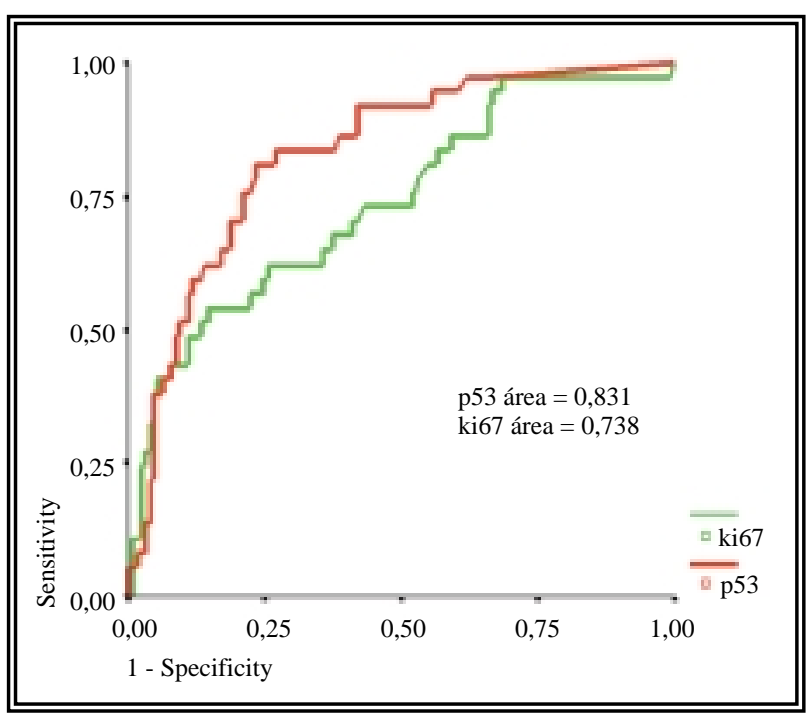

FIGURA 6. Curvas ROC DE p53 y ki67 para la progresión (área p53: 0,831 \pm 0,037; área p53: 0,738 $\pm 0,048$ ).

modo que a priori debería ser un buen marcador de progresión. Los resultados de nuestro estudio muestran que la expresión de ki67 >27\% predice la progresión de forma independiente. Estos resultados coinciden con los obtenidos por Liukkonen et $\mathrm{al}^{14}$. Pfister et $\mathrm{al}^{8} \mathrm{y}$ Zlotta et $\mathrm{al}^{17}$, por el contrario, no observan una relación significativa entre la expresión de ki67 y la progresión tumoral.

El punto de corte considerado para la expresión de p53 varía considerablemente en los estudios revisados, oscilando entre $>0 \%$ y el $40 \%$. Existen también diferencias en la inmunorreacti- vidad de los diferentes anticuerpos monoclonales empleados, siendo los clones DO7 y BP53-12 los que parecen más apropiados para realizar estudios inmunohistoquímicos, ya que con estos clones se obtienen un mayor porcentaje de núcleos teñidos, una mayor intensidad de tinción y una adecuada eliminación de la tinción inespecífica de fondo ${ }^{3}$.

El estudio inmunohistoquímico de ki67 plantea los mismos problemas que el de p53. La única diferencia es que en el caso de ki67 sólo existen dos anticuerpos monoclonales comercialmente disponibles (ki67 y MIB-1). La efectividad de ambos clones es similar ${ }^{8}$, aunque el porcentaje de inmunotinción varía ligeramente. El punto de corte utilizado para efectuar el análisis es bastante variable, como en el caso de p53, oscilando en los estudios revisados entre $>0 \%$ y $32 \%$.

En nuestro estudio hemos dividido las variables p53 y ki67 en cuartiles, con el propósito de obtener una mayor estratificación del riesgo. Este tipo de análisis nos ha permitido encontrar un pequeño grupo de pacientes, aquellos con p53 $>14 \%$, con un elevado riesgo de progresión.

El porcentaje de expresión de p53 es el principal factor pronóstico de progresión en nuestro estudio, observándose que los tumores con una expresión de p53 $>14 \%$ tienen un riesgo de progresión 74 veces superior a aquellos con expresión de $\mathrm{p} 53 \leq 0,2 \%$. Resultados similares han sido obtenidos por otros autores como Schmitz-Dräger et $\mathrm{al}^{13}$, Lacombe et $\mathrm{al}^{18}, \mathrm{y}$ Serth et $\mathrm{al}^{19}$.

\section{CONCLUSIONES}

Nuestro estudio, y otros similares, muestra una excelente relación entre la expresión de p53 y la probabilidad de progresión. Por esta razón, creemos que la expresión inmunohistoquímica de p53 debería ser considerada como uno de los factores de riesgo más importantes en la progresión del CCT de vejiga en estadio T1. Creemos también que los tumores de la categoría $\mathrm{T} 1$, con una expresión elevada de p53 deberían ser considerados y tratados como tumores de alto riesgo, independientemente de la presencia de otros factores tradicionalmente asociados al alto riesgo, como la multifocalidad tumoral, el elevado grado histológico, la multirrecidiva y el CIS secundario, entre otros. 


\section{REFERENCIAS}

1. WITJES JA, KIEMENEY LALM, SCHAAFSMA HE, DEBRUYNE FMJ: The influence of review pathology on study outcome of a randomized multicentre superficial bladder cancer trial. Br J Urol 1994; 73: 172-176.

2. HERMANN GG, HORN T, STEVEN K: The influence of the level of lamina propria invasion and the prevalence of p53 nuclear accumulation on survival in stage T1 transitional cell bladder cancer. J Urol 1998; 159: 91-94.

3. LINDEN MD, NATHANSON SD, ZARBO RJ: Evaluation of anti-p53 antibody staining. Quality control and technical considerations. Appl Immunohistochem 1994; 2 (3): 218-224.

4. MOSTOFI FK, SOBIN LH, TORLANI H: Histological typing of urinary bladder tumors. En International Histological Classification of Tumours. № 10 : Geneva: World Health Organization, 1973.

5. HERMANEK P, HUTTER RVP, SOBIN LH: TNM Atlas. Illustrated guide to the TNM/pTNM classification of malignant tumours. Separata de la $4^{\mathrm{a}}$ Edición en Inglés. Berlin, Heidelberg: Springer-Verlag; 1997: 263-326.

6. ALLARD P, BERNARD P, FRADET Y, TÊTU B: The early clinical course of primary Ta and T1 bladder cancer: a proposed prognostic index. Br J Urol 1998; 81: $692-698$.

7. WITJES JA, KIEMENEY LALM, VERBEEK ALM, HEIJBROEK RP, DEBRUYNE FMJ: Random bladder biopsies and the risk of recurrent superficial bladder cancer: a prospective study in 1026 patients. World J Urol 1992; 10: 231-234.

8. PFISTER C, LACOMBE L, VEZINA MC, MOORE L, LARUE H, TETTU B, et al: Prognostic value of the proliferative index determined by ki-67 immunostaining in superficial bladder tumors. Hum Pathol 1999; 11: 1350-1355.

9. OJEA-CALVO A, PÉREZ-GARCÍA MD, RODRÍGUEZALONSO A, DOMÍNGUEZ-FREIRE F, ALONSORODRIGO A, RODRÍGUEZ IGLESIAS B, et al: Cáncer superficial de vejiga: ¿qué controles han de seguir y durante cuanto tiempo, los pacientes libres de tumor?. Actas Urol Esp 1998; 22: 210-214.

10. MILLÁN-RODRÍGUEZ F, CHÉCHILE-TONIOLO G, SALVADOR-BAYARRI J, PALOU J, VICENTERODRÍGUEZ J: Multivariate analysis of the prognostic factors of primary superficial bladder cancer. J Urol 2000; 163: 73-78.

11. KIEMENEY LALM, WITJES JA, HEIJBROEK RP, VERBEEK ALM, DEBRUYNE FMJ: Predictability of recurrent and progressive disease in individual patients with primary superficial bladder cancer. $J$ Urol 1993; 150: 60-64.
12. MILLÁN-RODRÍGUEZ F, CHECHILE-TONIOLO G, SALVADOR-BAYARRI J, HUGUET-PÉREZ J, VICENTE-RODRÍGUEZ J: Upper urinary tract tumors after primary superficial bladder tumors: prognostic factors and risk groups. JUrol 2000; 164: 1.183-1.187.

13. SCHMITZ-DRÄGER BJ, KUSHIMA M, GOEBELL P, JAX TW, GERHARZ CD, BÜLTEL H, et al: p53 and MDM2 in the development and progression of bladder cancer. Eur Urol 1997; 32: 487-493.

14. LIUKKONEN T, RAJALA P, RAITANEN M, RINTALA E, KAASINEN E, LIPPONEN P: Prognostic value of MIB-1 score, p53, EGFr, mitotic index and papillary status in primary superficial (Stage $\mathrm{pTa} / \mathrm{T} 1$ ) bladder cancer: a prospective comparative study. Eur Urol 1999; 36: 393-400.

15. EPSTEIN JI, AMIN MB, REUTER VR, MOSTOFI FK: The bladder consensus conference committee. The World Health Organization/International Society of Urologic Pathology consensus classification of urothelial (transitional cell) neoplasms of the urinary bladder. Am J Surg Pathol 1998; 22: 1.435-1.438.

16. GONTERO P, CASETTA G, ZITELLA A, BALLARIO R, PACCHIONI D, MAGNANI C, et al: Evaluation of p53 protein overexpression, ki67 proliferative activity and mitotic index as markers of tumour recurrence in superficial transitional carcinoma of the bladder. Eur Urol 2000; 38: 287-296.

17. ZLOTTA AR, NOEL JC, FAYT I, DROWART A, VAN VOOREN JP, HUYGEN K, et al: Correlation and prognostic significance of p53, p2 $1^{\mathrm{WAF} 1 / \mathrm{CIP} 1}$ and ki67 expression in patients with superficial bladder tumors treated with Bacillus Calmette-Guérin intravesical therapy. J Urol 1999; 161: 792-798.

18. LACOMBE L, DALBAGNI G, ZHANG ZF, CORDÓNCARDO C, FAIR WR, HERR HW, et al: Overexpression of p53 protein in a high-risk population of patients with superficial bladder cancer before and after Bacillus Calmette-Guérin therapy: correlation to clinical outcome. J Clin Oncol 1996; 14: 2646-2652.

19. SERTH J, KUCZYK MA, BOKEMEYER C, HERVATIN C, NAFE R, TAN HK, et al: p53 immunohistochemistry as an independent prognostic factor for superficial cell carcinoma of the bladder. Br J Cancer 1995; 71: 201-205.

Dr. A. Rodríguez Alonso

Servicio de Urología

Hospital Arquitecto Marcide

Ctra. San Pedro de Leixa, s/n

15405 El Ferrol (A Coruña)

(Trabajo recibido el 13 de Junio 2002) 